Pacific Journal of 


\section{THE INTEGRATION OF A LIE ALGEBRA REPRESENTATION}

\section{J. TITS AND L. WAELBROECK}

Let $u: G \rightarrow A$ be a differentiable representation of a Lie group into a $b$-algebra. The differential $u_{0}=d u_{e}$ of $u$ at the neutral element $e$ of $G$ is a representation of the Lie algebra $g$ of $G$ into $A$. Because a Lie group is locally the union of one-parameter subgroups and since the infinitesimal generator of a differentiable (multiplicative) sub-semi-group of $A$ determines this sub-semi-group, the representation $u_{0}$ determines $u$ if $G$ is connected.

We shall be concerned with the converse: given a representation $u_{0}$ of $\mathrm{g}$, when can it be obtained by differentiating a representation $u$ of $G$ ? We shall assume $G$ connected and simply connected, which means that we are only interested in the local aspect of the problem.

Call $a \in A$ integrable if a differentiable $r: R \rightarrow A$ can be found such that $r(s+t)=r(s) r(t)$ and $r^{\prime}(0)=a$. We can only hope to integrate $u_{0}: \mathfrak{g} \rightarrow A$ to a differentiable $u: G \rightarrow A$ if $u_{0} x$ is integrable for all $x \in \mathfrak{g}$. We shall prove the

THEOREM. The set $\mathfrak{h}$ of all elements $x \in \mathfrak{g}$ such that $u_{0} x$ is integrable, is a Lie subalgebra of $\mathrm{g}$; the representation $u_{0}$ can be integrated to a representation $u: G \rightarrow A$ of the simply connected group $G$ if and only if $\mathfrak{h}=\mathfrak{g}$.

This result is "best possible" in the following sense:

Proposition 1. Given a real Lie algebra g and a subalgebra $\mathfrak{h}$, there exists a representation $u_{0}: \mathfrak{g} \rightarrow A$ of $\mathfrak{g}$ in a b-algebra $A$, so that

$$
\mathfrak{h}=\left\{x \in \mathfrak{g} \mid u_{0} x \text { is integrable }\right\} \text {. }
$$

As a consequence of the theorem, we have the following result: Let $x, y$ be two integrable elements of a $b$-algebra, and assume that the Lie algebra $g$ they generate is finite-dimensional. Then all elements of $\mathfrak{g}$ are integrable.

We cannot drop the assumption that $g$ is finite-dimensional. There exists a $b$-algebra which contains integrable elements $x, y$ such that neither $x+y$ nor $x y-y x$ is integrable.

Elementary properties of $b$-spaces and $b$-algebras can be found in [2] or [3]. Differentiable mappings into such spaces are investigated 
in [4]. The results we need about differentiable semi-groups are established in [5], [6]. Our results are related to, but different from, those of R. T. Moore [1].

2. We first prove Proposition 1 . Let $G$ be a Lie group having $\mathfrak{g}$ as Lie algebra and let $H$ be the subgroup of $G$ "generated" by $\mathfrak{h}$. Call $A$ the ring of distributions on $G$ whose support is compact and contained in $H$. The product in $A$ is the convolution. A subset $B$ of $A$ is bounded if $B$ is a bounded set of distributions with compact support, the union of the supports being relatively compact in $H$. Then, it is easily seen that the elements of $g$ whose image by the natural inclusion $u_{0}: \mathfrak{g} \rightarrow A$ are integrable, are precisely the elements of $\mathfrak{h}$. This completes the proof.

REMARK. If $H$ is simply connected, the algebra $A$ described above is the solution of a universal problem: every representation $u: \mathfrak{g} \rightarrow A^{\prime}$ of $\mathfrak{g}$ in a $b$-algebra $A^{\prime}$ such that $u \mathfrak{h}$ is integrable can be factorized in a unique way as $u=v \circ u_{0}$, where $v: A \rightarrow A^{\prime}$ is a morphism of $b$-algebras. An easy but somewhat technical modification of our definition of $A$ would provide a solution of this problem in general (for an arbitrary $H$ ); the reader will have no difficulty to figure it out.

3. Let $u$ be a differentiable mapping of a manifold $D$ into another manifold $D^{\prime}$ or into a $b$-space $E$. We denote by $d u(x ; \cdot)$ the derivative of $u$ at $x$, so that $d u(x, \xi)$ is a tangent vector to $D^{\prime}$ at $u x$ or an element of $E$ when $\xi$ is a tangent vector at $x \in D$. The chain rule says that if $D, D^{\prime}, D^{\prime \prime}$ are manifolds, if $E$ is a $b$-space and if $u: D \rightarrow D^{\prime}$, $v: D^{\prime} \rightarrow D^{\prime \prime}$ or $D^{\prime} \rightarrow E$ are differentiable mappings, then

$$
d(v \circ u)(x ; \xi)=d v(u x ; d u(x ; \xi)) \text {. }
$$

Let $G$ be a Lie group whose neutral element will be denoted by $e$ and let $g$ be its Lie algebra. If $x, y \in G$ and if $\xi$ is a tangent vector at $x$, then $y \xi$ and $\xi y$ will be the tangent vectors at $y x, x y$ respectively obtained by translating $\xi$ to the left or to the right. We shall denote by $\pi: G \times G \rightarrow G$ the product mapping $(\pi(x, y)=x y)$, by $i: G \rightarrow G$ the inverse mapping $\left(i(x)=x^{-1}\right)$, by $A d: G \rightarrow$ Aut $g$ the adjoint representation $\left(\operatorname{Ad} x \cdot \xi=x \xi x^{-1}\right)$ and by $a d$ the derivative of $A d$ at $e(a d \xi \cdot \eta=$ $[\xi, \eta])$. We have

$$
\begin{gathered}
d \pi(x, y ; \xi, \eta)=x \eta+\xi y ; \\
d i(x ; \xi)=-x^{-1} \cdot \xi \cdot x^{-1} .
\end{gathered}
$$

Let $H$ be a Lie group, let $A$ be a $b$-algebra and let $u$ denote 
either a Lie group homomorphism $G \rightarrow H$ or a differentiable mapping $G \rightarrow A$ which is a homomorphism of $G$ in the multiplicative group of $A$. Finally, set $u_{0}=d u(e ; \cdot): \mathfrak{g} \rightarrow \mathfrak{h}=$ Lie $H$ or $\mathfrak{g} \rightarrow A$ accordingly. Then

$$
d u(x ; \xi)=u(x) u_{0}\left(x^{-1} \xi\right)=u_{0}\left(\xi x^{-1}\right) u(x)
$$

In particular

$$
d A d(x ; \xi)=A d x \cdot \operatorname{ad}\left(x^{-1} \xi\right)=\operatorname{ad}\left(\xi x^{-1}\right) \cdot A d x .
$$

4. Let $A$ be a $b$-algebra and $A^{*}$ be the set of its invertible elements. A mapping $u: D \rightarrow A^{*}$ will be called differentiable if both $x \rightarrow u(x)$ and $x \rightarrow u(x)^{-1}$ are differentiable mappings.

It is not difficult to construct differentiable $A$-valued mappings which are $A^{*}$-valued but are not differentiable $A^{*}$-valued mappings.

Consideration of the resolvent identity

$$
a^{-1}-b^{-1}=-a^{-1}(a-b) b^{-1}
$$

and standard proofs show that a differentiable mapping $u: D \rightarrow A^{*}$ with values in $A^{*}$ is a differentiable $A^{*}$-valued mapping in the above sense if and only if $u^{-1}: D \rightarrow A$ is locally bounded. It turns out that

$$
d u^{-1}(x ; \xi)=-u^{-1}(x) \cdot d u(x ; \xi) \cdot u^{-1}(x) .
$$

5. From now on, $G$ will be a connected, simply connected Lie group, $\mathfrak{g}$ will be its Lie algebra, $A$ a $b$-algebra and $u_{0}: \mathfrak{g} \rightarrow A$ a representation. A differentiable submanifold $D$ of $G$ is called right (resp. left) integrable for $u_{0}$ if a differentiable $u: D \rightarrow A^{*}$ exists such that the equation (7) (resp. (8)) holds:

$$
\begin{aligned}
& d u(x ; \xi)=u_{0}\left(\xi \cdot x^{-1}\right) u(x) ; \\
& d u(x ; \xi)=u(x) u_{0}\left(x^{-1} \cdot \xi\right) .
\end{aligned}
$$

It will follow from Proposition 2 that the representation $u_{0}$ is integrable in the sense of $\S 1$ if and only if the manifold $G$ itself is right or left integrable; therefore the terminology. We note that, if $u$ satisfies (7), then

$$
d u^{-1}(x ; \xi)=-u^{-1}(x) u_{0}\left(\xi \cdot x^{-1}\right) .
$$

A right translate of a right integrable manifold is right integrable. If $u$ satisfies (7), so does $a u$ for every $a \in A^{*}$.

Lemma 1. Let $D$ be connected, right integrable, containing e, and let $u$ be a solution of $(7)$ such that $u(e)=1$. Then 


$$
u_{0}\left(x \xi x^{-1}\right)=u(x) u_{0}(\xi) u(x)^{-1}
$$

for all $x \in D$ and $\xi \in \mathrm{g}$.

It suffices to show that if $\varphi: D \rightarrow A$ is defined by

$$
\varphi(x)=u(x)^{-1} u_{0}\left(x \xi x^{-1}\right) u(x),
$$

then $d \varphi=0$, and this follows from a straightforward computation using (7), (9), (5) and the fact that $u_{0}: \mathfrak{g} \rightarrow A$ is a homomorphism of Lie algebras.

Lemma 2. If $D$ is connected, right integrable and contains $e$, it is also left integrable. Furthermore, the solution $u$ of (7) such that $u(e)=1$ is also a solution of (8).

This is clear since, by (10),

$$
u(x) u_{0}\left(x^{-1} \xi\right)=u_{0}\left(x \cdot x^{-1} \xi \cdot x^{-1}\right) u(x)=u_{0}\left(\xi x^{-1}\right) u(x) .
$$

In view of Lemma 2 , it is now meaningful to say that a manifold containing $e$ is integrable.

6. Let $D, D^{\prime}$ be two differentiable manifolds. The rank $r_{x}$ of a differentiable mapping $u: D \rightarrow D^{\prime}$ at a point $x \in D$ is the dimension of the image of the derivative $d u(x ; \cdot)$. We recall that $r_{x}$ is upper semi-continuous as a function of $x$. The mapping $u$ is said to be regular at $x$ if $r_{x}$ is constant in a neighborhood of $x$; in that case, there exists a neighborhood $U$ of $x$, a submanifold $D^{\prime \prime}$ of $D^{\prime}$, a manifold $E$ and a diffeomorphism $u^{\prime}: U \rightarrow D^{\prime \prime} \times E$, so that $\left.u\right|_{U}=p_{D^{\prime \prime}} \circ u^{\prime}$ where $p_{D^{\prime \prime}}$ denotes the projection of $D^{\prime \prime} \times E$ of its first factor.

Lemma 3. For $i=1,2$, let $D_{i}$ be an integrable submanifold of $G$ containing $e$, and let $u_{i}: D_{i} \rightarrow A$ be a solution of (7) mapping $e$ on 1 . Assume that the product mapping $D_{1} \times D_{2} \rightarrow G$ is regular at $(e, e)$. Then, one can find neighborhoods $D_{1}^{\prime}, D_{2}^{\prime}$ of $e$ in $D_{1}, D_{2}$ respectively, so that $D=D_{1}^{\prime} \cdot D_{2}^{\prime}$ is an integrable manifold and the relation

$$
u\left(x_{1} \cdot x_{2}\right)=u_{1}\left(x_{1}\right) \cdot u_{2}\left(x_{2}\right) \quad\left(x_{i} \in D_{i}^{\prime}\right)
$$

defines a mapping $u: D \rightarrow A$ which is a solution of (7).

Put $v\left(x_{1}, x_{2}\right)=u_{1}\left(x_{1}\right) u_{2}\left(x_{2}\right)$, differentiate and apply (7), (10) and (2). This yields

$$
d v\left(x_{1}, x_{2} ; \xi_{1}, \xi_{2}\right)=u_{0}\left(d \pi\left(x_{1}, x_{2} ; \xi_{1}, \xi_{2}\right) x_{2}^{-1} x_{1}^{-1}\right) v\left(x_{1}, x_{2}\right) .
$$

In particular, $d v=0$ whenever $d \pi=0$. This, the regularity assump- 
tion and the implicit function theorem imply the existence of a function $u$ satisfying (11) locally. In view of (12), this function is locally a solution of (7).

7. Our main theorem is an immediate consequence of the

Proposition 2. Let $D$ be an integrable submanifold of $G$ of maximum dimension containing $e$ and let $u: D \rightarrow A$ be the solution of (7) with $u(e)=1$. Then $D$ is a local subgroup, $u$ is a local homomorphism of $D$ into $A^{*}$ and $D$ contains locally every integrable submanifold of $G$ containing $e$.

We first show that

(*) $^{*}$ if $D^{\prime}$ is any integrable submanifold of $G$ containing $e$, the tangent space to $D^{\prime}$ at $e$ is contained in that of $D$.

Assume the contrary. Then there exists a neighborhood $U$ of $(e, e)$ in $D \times D^{\prime}$ such that, for every $\left(x, x^{\prime}\right) \in U$, the tangent space to $x^{-1} D$ at $e$ does not contain that to $D^{\prime} x^{\prime-1}$. Let $\left(f, f^{\prime}\right) \in U$ be a point where the product mapping $D \times D^{\prime} \rightarrow D \cdot D^{\prime}$ is regular (one knows that the set of those points is dense). Then, by Lemma 3, there exist neighborhoods $E$ of $f$ in $D$ and $E^{\prime}$ of $f^{\prime}$ in $D^{\prime}$ such that $f^{-1} E E^{\prime} f^{\prime-1}$ is an integrable manifold, which is obviously of dimension greater than that of $D$, in contradiction to the maximality assumption.

It follows from $\left({ }^{*}\right)$ that the tangent space to $D$ at any one of its points, say $x$, is a translate of its tangent space at $e$ (take $\left.D^{\prime}=x^{-1} D\right)$. This ensures that $D$ is a local group.

Since $D$ is a local group, the product mapping $D \times D \rightarrow D$ is regular in $(e, e)$. It then follows from Lemma 3 that there exist a neighborhood $U$ of $(e, e)$ in $D \times D$ and a function $v$ defined in a neighborhood of $e$ in $D$ so that

$$
v\left(x_{1} x_{2}\right)=u\left(x_{1}\right) u\left(x_{2}\right)
$$

for $\left(x_{1}, x_{2}\right) \in U$. But then, for points $x_{1}, x_{2}$ close enough to $e$, we have

$$
u\left(x_{1}\right) u\left(x_{2}\right)=v\left(x_{1} x_{2} \cdot e\right)=u\left(x_{1} x_{2}\right) \cdot u(e)=u\left(x_{1} x_{2}\right),
$$

and $u$ is a local representation.

Finally, if $D^{\prime}$ is integrable (right or left), it follows from (8) that the tangent space to $D^{\prime}$ at any one of its points is contained in a translate of the tangent space to $D$ at $e$. If $e \in D^{\prime}$, this implies that $D^{\prime}$ is locally (at $e$ ) contained in $D$. 


\section{BIBLIOGRAPHY}

1. R. T. Moore, Exponentiation of operator Lie algebras on Banach spaces, Bull. Amer. Math. Soc. 71 (1965), 903-908.

2. L. Waelbroeck, Etude spectrale des algèbres complètes, Acad. Roy. de Belgique. Mém. Cl. des Sc., 1960.

3. - Some theorems about bounded structures, J. of Functional Analysis 1 (1967), 392-408.

4. —, Differentiable mappings into b-spaces, J. of Functional Analysis 1 (1967), $409-418$.

5. — Les semi-groupes différentiables, Deuxième Colloque d'Analyse fonctionnelle, 97-103. C.B.R.M. Librairie Universitaire Louvain, 1964.

6. — Differentiability of Hölder continuous semi-groups (to appear in Proc. Amer. Math. Soc.).

Received November 27, 1967.

UNIVERSITY OF BONN

UNiversity of Brussels and UNiversity of California, Los ANgeles 


\section{PACIFIC JOURNAL OF MATHEMATICS}

\section{EDITORS}

\section{H. ROYDEN}

Stanford University

Stanford, California

R. R. Phelps

University of Washington

Seattle, Washington 98105

\section{J. DugundJI}

Department of Mathematics

University of Southern California

Los Angeles, California 90007

RICHARD ARENS

University of California

Los Angeles, California 90024

\section{ASSOCIATE EDITORS}
E. F. BeCKENBACH
B. H. NeumanN
F. WOLF
K. YosIDA

\section{SUPPORTING INSTITUTIONS}

\author{
UNIVERSITY OF BRITISH COLUMBIA \\ CALIFORNIA INSTITUTE OF TECHNOLOGY \\ UNIVERSITY OF CALIFORNIA \\ MONTANA STATE UNIVERSITY \\ UNIVERSITY OF NEVADA \\ NEW MEXICO STATE UNIVERSITY \\ OREGON STATE UNIVERSITY \\ UNIVERSITY OF OREGON \\ OSAKA UNIVERSITY \\ UNIVERSITY OF SOUTHERN CALIFORNIA
}

\author{
STANFORD UNIVERSITY \\ UNIVERSITY OF TOKYO \\ UNIVERSITY OF UTAH \\ WASHINGTON STATE UNIVERSITY \\ UNIVERSITY OF WASHINGTON \\ AMERICAN MATHEMATICAL SOCIETY \\ CHEVRON RESEARCH CORPORATION \\ TRW SYSTEMS \\ NAVAL WEAPONS CENTER
}

Mathematical papers intended for publication in the Pacific Journal of Mathematics should be in typed form or offset-reproduced, double spaced with large margins. Underline Greek letters in red, German in green, and script in blue. The first paragraph or two must be capable of being used separately as a synopsis of the entire paper. It should not contain references to the bibliography. Manuscripts, in duplicate if possible, may be sent to any one of the four editors. All other communications to the editors should be addressed to the managing editor, Richard Arens, University of California, Los Angeles, California 90024.

Each author of each article receives 50 reprints free of charge; additional copies may be obtained at cost in multiples of 50 .

The Pacific Journal of Mathematics is published monthly. Effective with Volume 16 the price per volume (3 numbers) is $\$ 8.00$; single issues, $\$ 3.00$. Special price for current issues to individual faculty members of supporting institutions and to individual members of the American Mathematical Society: $\$ 4.00$ per volume; single issues $\$ 1.50$. Back numbers are available.

Subscriptions, orders for back numbers, and changes of address should be sent to Pacific Journal of Mathematics, 103 Highland Boulevard, Berkeley 8, California.

Printed at Kokusai Bunken Insatsusha (International Academic Printing Co., Ltd.), 7-17, Fujimi 2-chome, Chiyoda-ku, Tokyo, Japan.

PUBLISHED BY PACIFIC JOURNAL OF MATHEMATICS, A NON-PROFIT CORPORATION

The Supporting Institutions listed above contribute to the cost of publication of this Journal, but they are not owners of publishers and have no responsibility for its content or policies. 


\section{Pacific Journal of Mathematics}

\section{Vol. 26, No. $3 \quad$ BadMonth, 1968}

Leonard Asimow, Universally well-capped cones ................. 421

Lawrence Peter Belluce, William A. Kirk and Eugene Francis Steiner,

Normal structure in Banach spaces ..................... 433

William Jay Davis, Bases in Hilbert space.................... 441

Larry Lee Dornhoff, p-automorphic p-groups and homogeneous

algebras..................................... 447

William Grady Dotson, Jr. and W. R. Mann, A generalized corollary of the

Browder-Kirk fixed point theorem ....................... 455

John Brady Garnett, On a theorem of Mergelyan ................. 461

Matthew Gould, Multiplicity type and subalgebra structure in universal

algebras............................................. 469

Marvin D. Green, A locally convex topology on a preordered space . . . . . 487

Pierre A. Grillet and Mario Petrich, Ideal extensions of semigroups . . . . . . 493

Kyong Taik Hahn, A remark on integral functions of several complex

variables ... . . . . . . . . . . . . . . . . . . . . . . . . . . . . . . . . . . . 509

Choo Whan Kim, Uniform approximation of doubly stochastic

operators..................................... 515

Charles Alan McCarthy and L. Tzafriri, Projections in $\mathscr{L}_{1}$ and

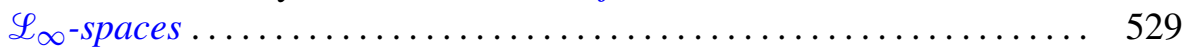

Alfred Berry Manaster, Full co-ordinals of RETs ................ 547

Donald Steven Passman, $p$-solvable doubly transitive permutation

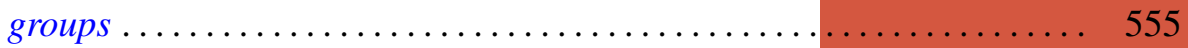

Neal Jules Rothman, An $L^{1}$ algebra for linearly quasi-ordered compact

semigroups ....................................... 579

James DeWitt Stein, Homomorphisms of semi-simple algebras .......... 589

Jacques Tits and Lucien Waelbroeck, The integration of a Lie algebra

representation ...............................

David Vere-Jones, Ergodic properties of nonnegative matrices. II ........ 601

Donald Rayl Wilken, The support of representing measures for $R(X) \ldots \ldots 621$

Abraham Zaks, Simple modules and hereditary rings .... . . 